\title{
两种基于联萗酚的手性聚醚诱导苯乙炔对醛的对映选择性加成反应
}

\author{
禹曾达 \\ 刘资 \\ 张令君* \\ 阳年发* \\ (湘潭大学化学学院 环境友好化学与应用省部共建教育部重点实验室 湘潭 411105)
}

摘要 合成了两种带联䒺酚侧基的手性聚醚, 并将其应用于诱导苯乙炔对醛的不对称加成反应中. 产物具有较好的对 映选择性, ee 值最高达 $95 \%$. 这种聚醚可以被简单的回收重复使用 6 次且催化活性基本不变.

关键词 环氧氯丙烷; 聚醚; 联芸酚; 苯乙炔

\section{Synthesis of Two 1,1'-Bi-2-naphthol-Based Chiral Polyethers for the Enantioselective Addition of Phenylacetylene to Aldehydes}

\author{
Yu, Zengda Liu, Feng Zhang, Lingjun * Yang, Nianfa* \\ (Key Laboratory of Environmentally Friendly Chemistry and Applications of Ministry of Education, \\ College of Chemistry, Xiangtan University, Hunan 411105)
}

\begin{abstract}
Two polyethers derived from bi-2-naphthol (BINOL) were synthesized and its asymmetric inducement in the addition of phenylacetylene to aldehydes was studied. The addition products have good enantioselectivity with up to $95 \%$ enantiomeric excess (ee). Furthermore, the polyether could be conveniently recovered and reused at least 6 times without loss of catalytic ability.

Keywords epichlorohydrin; polyether; bi-2-naphthol; phenylacetylene
\end{abstract}

不对称催化是现代有机合成最具挑战性的领域之 一, 一个成功的不对称反应应该具备以下三个特点: (1) 产物应该具有较高的 $e e$ 值(对映体过量值); (2)手性配体 容易制备和再利用; (3)能够根据需求制备 $R$ 和 $S$ 两种构 型的产品. 虽然现在已经有很多优异的手性小分子催化 剂被合成出来 ${ }^{[1 ~ 8]}$, 但由于大多数的手性小分子催化剂 是非常昂贵并且难以和催化产物分离, 导致催化成本很 高. 因此, 科学家们对手性催化剂的回收利用越来越感 兴趣 ${ }^{[920]}$. 相对于小分子催化剂, 聚合物催化剂在很多 的不良溶剂中能够完全沉降, 从而到达很好的分离. 因 此, 设计和合成聚合物催化剂是解决催化剂回收利用的 有效途径之一. 我们课题组多年来一直致力于手性聚合 物催化剂的研究, 并且在许多的不对称反应中取得了显 著的进展 ${ }^{[21 ~ 28] . ~}$

众所周知, 手性炔醇是许多手性化合物非常重要的 前体 ${ }^{[29 ~ 35]}$, 而炔基锌对醛的不对称加成是制备手性炔 醇的有效途径之一 ${ }^{[36 ~ 51]}$. 虽然已经有大量的手性小分
子催化剂能很好地催化制备手性炔醇 ${ }^{[36 ~ 51]}$, 但是仅仅 只有少量可以回收利用的聚合物催化剂被报道 ${ }^{[2]}$. 因 此, 基于我们前期合成的联菜酚衍生的聚醚 Poly-A 和 Poly-B(图 1)在对映选择性硼烷还原潜手性酮的优异表 现 ${ }^{[26,27]}$. 我们再次尝试将它们应用于诱导苯乙炔对醛的 不对称加成中. 令人欣慰的是, 我们获得的手性炔丙基 醇的 $e e$ 值最高达 $95 \%$, 而且这种聚醚还可以被回收利 用多次且催化活性没有明显降低.<smiles>OCC(O)COCC1=CC=c2cccc(O)c2=C2C(O)=CC=c3ccccc3=C12</smiles><smiles>OCC(O)COCc1ccc2c(-c3c(O)ccc4ccccc34)c(O)ccc2c1</smiles>

图 1 手性聚合物配体 Poly-A 和 Poly-B 的结构

Figure 1 Structures of the chiral polymer ligands Poly-A and Poly-B

\footnotetext{
* Corresponding authors. E-mail: dudu8394@163.com; nianfayang@xtu.edu.cn Received December 25, 2016; revised March 21, 2017; published online April 21, 2017. Project supported by the Higher Education Doctoral Science Foundation of China (No. 20134301110004). 高等学校博士学科点专项科研基金(No. 20134301110004)资助项目.
} 


\section{1 结果与讨论}

\section{1 带联荎侧基的大分子配体的合成与表征}

根据之前的研究 ${ }^{[26]}$, 我们合成了 $(S, S)$-6-(2,3-环氧 丙氧基)甲基-2,2'-二(甲氧基甲氧基)-1, $1^{\prime}$-联萗 (8a) 和 $(S$, $R$ )-6-(2,3-环氧丙氧基)甲基-2,2'-二(甲氧基甲氧基)-1,1'联萗 $(\mathbf{8 b})$, 并将其以氢氧化钾 $(\mathrm{KOH})$ 为引发剂进行了阴 离子聚合. 然后在盐酸作用下脱去甲氧基甲氧基得到相 应的聚醚配体 Poly-A 和 Poly-B. 具体合成路线见 Scheme 1. Poly-A: 产率 $85 \%,[\alpha]_{365}^{20}-342.4$ (c 0.05 , THF); $M_{\mathrm{n}}=5.2 \times 10^{3} ; \mathrm{PDI}=M_{\mathrm{w}} / M_{\mathrm{n}}=2.02$. Poly-B: 产率 $82 \%,[\alpha]_{365}^{20}-296.7$ (c 0.05 , THF); $M_{\mathrm{n}}=5.6 \times 10^{3}$; PDI $=$ $M_{\mathrm{w}} / M_{\mathrm{n}}=2.41$.

\section{2 苯乙炔与醛的不对称加成反应}

\subsection{1 苯乙炔与芳基醛反应的条件优化}

参考蒲林的研究 ${ }^{[36]}$, 我们将苯乙炔与醛的加成反 应分两个步骤: (1)苯乙炔和二甲基锌制备炔基锌. (2)依 次加入配体 Poly-A 或 Poly-B、钛酸异丙酯、醛. 以 $n$ (苯 乙炔) $: n$ (二甲基锌) $: n$ (苯甲醛) $=4: 4: 1$ 为模型反应
进行了条件探索(表 1). 首先, 对配体 Poly-A 和 Poly-B 进行了篎选(表 1, Entries 1,2), 当配体由 Poly-A 换为 Poly-B 时，反应的对映异构体选择性有所下降(Entry 2). 接着考察了溶剂对反应的影响(表 1 , Entries 1,3 6), 当 溶剂为四氢呋喃时, 产物的产率和 $e e$ 值最好, 分别为 94\%和 79\% (Entry 1). 然后考察了温度对反应的影响(表 1 , Entries 1,7), 当把温度降到 $0{ }^{\circ} \mathrm{C}$ 时, 虽然 $e e$ 值 $(85 \%)$ 有所提高, 但是产率急剧下降到 30\% (Entry 7), 因此选 择 $25{ }^{\circ} \mathrm{C}$ 为最佳反应温度. 随后对铁酸异丙酯的用量考 察表明(Entries 1,8,9), 当钛酸异丙酯用量 $100 \%$ 时, 得 到 91\%产率和 $85 \%$ ee (Entry 8). 最后考察了配体的用 量, 发现反应产率会随着配体用量增加而降低(Entries $8,10,11)$, 当配体用量 $20 \%$ 时, 产物的 $e e$ 值最高 $90 \%$ 和 产率为 $87 \%$ (Entry 10). 延长反应时间至 $24 \mathrm{~h}$, 产率得到 了提高(Entry 12). 最终选定的最优条件为 $25{ }^{\circ} \mathrm{C}$ 下 $\mathrm{THF}$ 为溶剂反应 $24 \mathrm{~h}: n$ (醛) $: n\left[\mathrm{Ti}(\mathrm{O}-i-\mathrm{Pr})_{4}\right]: n\left(\mathrm{Me}_{2} \mathrm{Zn}\right)$ : $n$ (苯乙炔 $) ： n$ (配体 Poly-A) $=1.0 ： 1.0: 4.0 ： 4.0 ０ .2$.

\subsection{2 底物拓展}

按照上述的最优条件，我们将不同的醛应用于该反

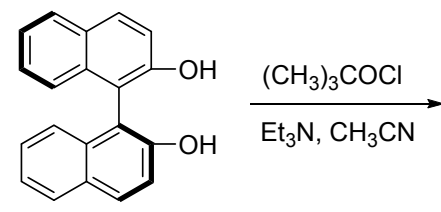

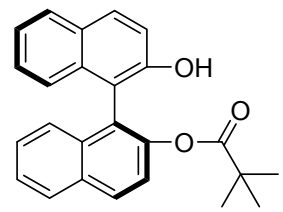

2

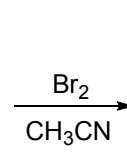

$\mathrm{Br}$<smiles>Oc1ccc2ccccc2c1-c1c(O)ccc2cc(Br)ccc12</smiles>

4

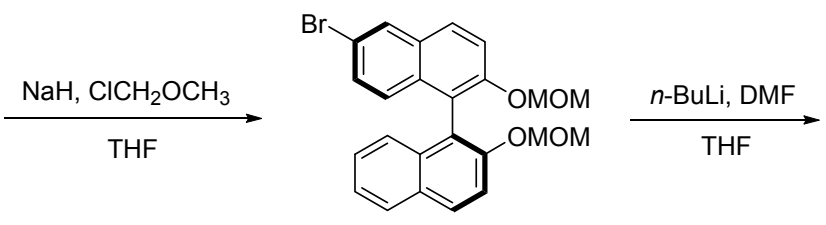

5

$\mathrm{OHC}$

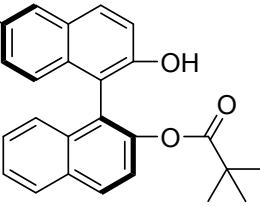

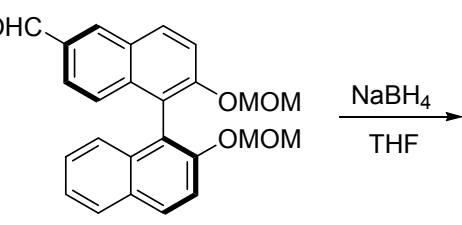

6<smiles>COc1ccc2ccccc2c1-c1c(OC)ccc2ccccc12</smiles>

7

(1) $\mathrm{KOH}$, xylene, $120^{\circ} \mathrm{C}$

(2) $\mathrm{HCl}, \mathrm{THF}$, r.t.<smiles></smiles>

Poly-A

(1) $\mathrm{KOH}$, xylene, $120^{\circ} \mathrm{C}$ (2) $\mathrm{HCl}, \mathrm{THF}$, r.t.

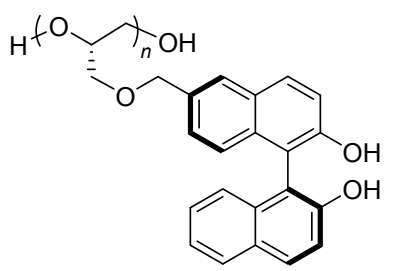

Poly-B

图式 1 聚合物 Poly-A 和 Poly-B 的合成 Scheme 1 Synthetic routes of Poly-A and Poly-B 
表 1 聚合物 Poly-A 或 Poly-B 催化苯乙炔与苯甲醛的不对称加成反应条件优化 ${ }^{a}$

Table 1 Optimization of asymmetric reaction of phenylacetylene with benzaldehyde catalyzed by Poly-A or Poly-B

$$
\mathrm{Ph}=\frac{\mathrm{Me}_{2} \mathrm{Zn}}{\operatorname{Step~} 1}[\mathrm{Ph}=\mathrm{ZnMe}] \stackrel{\mathrm{Ph}-\mathrm{CHO}}{\stackrel{\text { Ligand, Ti(O-i-Pr})_{4}}{\longrightarrow}}
$$

\begin{tabular}{|c|c|c|c|c|c|c|c|}
\hline \multirow{2}{*}{ Entry } & \multicolumn{2}{|c|}{ Conditions for step 2} & \multirow{2}{*}{$\mathrm{Ti}(\mathrm{O}-i-\mathrm{Pr})_{4} / \mathrm{mol} \%$} & \multirow{2}{*}{ Ligand (mol\%) } & \multirow{2}{*}{ Yield $^{b} / \%$} & \multirow{2}{*}{$e e^{c} / \%$} & \multirow{2}{*}{ Config. ${ }^{d}$} \\
\hline & Solvent & $T /{ }^{\circ} \mathrm{C}$ & & & & & \\
\hline 1 & THF & 25 & 50 & Poly-A (10) & 94 & 79 & $R$ \\
\hline 2 & THF & 25 & 50 & Poly-B (10) & 90 & 67 & $R$ \\
\hline 3 & Toluene & 25 & 50 & Poly-A (10) & 90 & 38 & $R$ \\
\hline 4 & $\mathrm{Et}_{2} \mathrm{O}$ & 25 & 50 & Poly-A (10) & 87 & 18 & $R$ \\
\hline 5 & DCE & 25 & 50 & Poly-A (10) & 89 & 71 & $R$ \\
\hline 6 & DCM & 25 & 50 & Poly-A (10) & 92 & 47 & $R$ \\
\hline 7 & THF & 0 & 50 & Poly-A (10) & 30 & 85 & $R$ \\
\hline 8 & THF & 25 & 100 & Poly-A (10) & 91 & 85 & $R$ \\
\hline 9 & THF & 25 & 150 & Poly-A (10) & 84 & 82 & $R$ \\
\hline 10 & THF & 25 & 100 & Poly-A (20) & 87 & 90 & $R$ \\
\hline 11 & THF & 25 & 100 & Poly-A (30) & 82 & 88 & $R$ \\
\hline $12^{e}$ & THF & 25 & 100 & Poly-A (20) & 90 & 91 & $R$ \\
\hline
\end{tabular}

${ }^{a}$ Conditions for step 1: $2 \mathrm{~mL}$ of toluene, reflux $5 \mathrm{~h}$; phenylacetylene: $2.0 \mathrm{mmol} ; \mathrm{Me}_{2} \mathrm{Zn}: 2.0 \mathrm{mmol}$; Conditions for step 2: $8 \mathrm{~mL}$ of solvent; benzaldehyde: $0.5 \mathrm{mmol}$; reaction time: $8 \mathrm{~h} .{ }^{b}$ Isolated yields. ${ }^{c}$ Determined by HPLC with a Chiralcel OD-H column. ${ }^{d}$ The absolute configurations of the products were determined by comparison to the literature data ${ }^{[39]}$. ${ }^{e}$ Reaction time: $24 \mathrm{~h}$.

应中来, 得到了从 $75 \% \sim 95 \% \mathrm{ee}$ 值(表 2). 当底物是苯 甲醛(Entry 1) 时产物 $e e$ 值为 $94 \%$, 当底物为带有给电子 取代基 $\mathrm{OCH}_{3}$ 时产物的 $e e$ 值有所降低(Entries 2,3), 其中 邻位取代 $\mathrm{OCH}_{3}$ 的更低一些(Entry 2); 当底物为带有吸 电子取代基的对氯苯甲醛(Entry 4)、对溴苯甲醛(Entry 5)、对氟苯甲醛(Entry 6)、对硝基苯甲醛(Entry 7)时产物 都有较高的 $e e$ 值, 分别达到 $90 \%, 85 \%, 89 \%$ 和 $95 \%$; 间 位取代的 $\mathrm{NO}_{2}$ 比对位取代的 $\mathrm{NO}_{2}$ 表现出明显要低的对 映选择性(Entry 8)；1-菜甲醛的加成产物(Entry 9) ee 值 比 2-䒺甲醛(Entry 10)要低, 我们认为是由于二者空间 位阻差异引起的; 值得一提的是, 当底物换成苯丙醛 (Entry 11)和正庚醛(Entry 12)时, 配体 Poly-A 依然表现 出不错的手性诱导作用, 它们的 $e e$ 值分别有 $80 \%$ 和 $75 \%$, 且收率分别高达 92\% 和 95\%. 这表明配体 Poly-A 具有良好的底物适用性.

\subsubsection{Poly-A 的回收重复利用}

相对于联䒺酚, 联䒺酚衍生的聚醚在一些有机溶剂 如甲醇、乙醇中的溶解度很差, 使其容易通过一个简单 的沉降方法予以回收. 我们测试聚合物 Poly-A 的循环 再利用效率. Poly-A 参与一次催化过程之后, 将回收的 聚合物 Poly-A (1.0 g)溶于 $20 \mathrm{~mL}$ 二氯甲烷中, 加 $1 \mathrm{~mL}$ 浓盐酸, 搅拌 $1 \mathrm{~h}$ 后分出有机层. 减压除去大部分溶剂 后倒入 $100 \mathrm{~mL}$ 甲醇中, 析出固体, 抽滤, 将滤渣用甲醇 洗涤两次. $40{ }^{\circ} \mathrm{C}$ 真空干燥 $8 \mathrm{~h}$ 即得回收配体, 配体回收 利用 6 次的收率分别为 $96 \% 、 98 \% 、 99 \% 、 97 \% 、 98 \%$ 、 $98 \%$. 通过 6 次循环使用结果(表 3)可以看出, 催化产物 的 $e e$ 值和产率没有发生明显变化.
表 2 聚合物 Poly-A 催化苯乙炔与各种醛的不对称加成反应 ${ }^{a}$ Table 2 Enantioselective addition of phenylacetylene to various aldehydes catalyzed by Poly-A

$$
\begin{aligned}
& \mathrm{Ph}=\frac{\mathrm{Me}_{2} \mathrm{Zn}}{\operatorname{Step~} 1}[\mathrm{Ph}=\mathrm{ZnMe}] \frac{\mathrm{Ri}(\mathrm{O}-\mathrm{i}-\mathrm{Pr})_{4} \text {, Ligand Poly-A }}{\text { Step } 2} \\
& \mathrm{OH} \\
& \mathrm{R}^{*} \approx \mathrm{Ph}
\end{aligned}
$$

\begin{tabular}{clccc}
\hline Entry & \multicolumn{1}{c}{$\mathrm{R}$} & Yield $^{b} / \%$ & $e e^{c} / \%$ & Config. $^{d}$ \\
\hline 1 & Phenyl & 92 & 91 & $R$ \\
2 & 2-Methoxyphenyl & 82 & 81 & $R$ \\
3 & 3-Methoxyphenyl & 83 & 86 & $R$ \\
4 & 4-Chlorophenyl & 90 & 90 & $R$ \\
5 & 4-Bromophenyl & 87 & 85 & $R$ \\
6 & 4-Fluorophenyl & 91 & 89 & $R$ \\
7 & 4-Nitrophenyl & 89 & 95 & $R$ \\
8 & 3-Nitrophenyl & 86 & 85 & $R$ \\
9 & 1-Naphthyl & 81 & 88 & $R$ \\
10 & 2- Naphthyl & 82 & 92 & $R$ \\
11 & 3-Phenethyl & 92 & 80 & $R$ \\
12 & Hexyl & 95 & 75 & $R$ \\
\hline
\end{tabular}

${ }^{a}$ Conditions for step 1: $2 \mathrm{~mL}$ of toluene, reflux $5 \mathrm{~h}$; phenylacetylene: 2.0 mmol; $\mathrm{Me}_{2} \mathrm{Zn}: 2.0 \mathrm{mmol}$; Conditions for step 2: reactions were carried out with $20 \mathrm{~mol} \%$ of Poly-A (as a BINOL monomer), $0.5 \mathrm{mmol}$ of aldehyde, 1.0 mmol of $\mathrm{Ti}(\mathrm{O}-i-\mathrm{Pr})_{4}$ in $8 \mathrm{~mL}$ of THF at $25{ }^{\circ} \mathrm{C}$ for $24 \mathrm{~h} .{ }^{b}$ Isolated yield. ${ }^{c}$ Determined by HPLC using a Chiralcel OD-H column. ${ }^{d}$ The absolute configurations of the products were determined by comparison to the literature data $^{[52]}$.

\section{2 结论}

以手性环氧氯丙烷和联菱酚为起始物合成了两种 环氧化合物, 并通过阴离子聚合以及脱 MOM 保护过程 
表 3 回收的 Poly-A 催化苯乙炔与苯甲醛的不对称加成反应 ${ }^{a}$ Table 3 Asymmetric reaction of phenylacetylene with benzaldehyde catalyzed by the recovered Poly-A

\begin{tabular}{rcccccc}
\hline Cycle & 1 & 2 & 3 & 4 & 5 & 6 \\
\hline Yield $^{b} \%$ & 92 & 91 & 90 & 91 & 89 & 91 \\
$e e^{c} / \%$ & 91 & 91 & 90 & 92 & 93 & 90 \\
\hline
\end{tabular}

${ }^{a}$ Conditions for step 1: $2 \mathrm{~mL}$ of toluene, reflux $5 \mathrm{~h}$; phenylacetylene: 2.0 $\mathrm{mmol} ; \mathrm{Me}_{2} \mathrm{Zn}$ : $2.0 \mathrm{mmol}$; Conditions for step 2: reactions were carried out with $20 \mathrm{~mol} \%$ of Poly-A (as a BINOL monomer), $0.5 \mathrm{mmol}$ of benzaldehyde, $1.0 \mathrm{mmol}$ of $\mathrm{Ti}(\mathrm{O}-i-\mathrm{Pr})_{4}$, in $8 \mathrm{~mL}$ of THF at $25{ }^{\circ} \mathrm{C}$ for $24 \mathrm{~h}^{\circ}{ }^{b}$ Isolated yield. ${ }^{c}$ Determined by HPLC using a Chiralcel OD-H column.

形成了对应的聚醚 Poly-A 和 Poly-B. 这两种聚合物被 用于诱导苯乙炔与醛的不对称加成反应, 获得了最高 $95 \%$ 的 $e e$ 值的对映选择性. 同时, 催化剂可以回收和重 复使用多次而其催化活性没有明显损失.

\section{3 实验部分}

\section{1 仪器与试剂}

${ }^{1} \mathrm{H}$ NMR 和 ${ }^{13} \mathrm{C}$ NMR 谱图使用德国 Bruker 公司的 $\mathrm{AV}-400$ 核磁共振仪(以 $\mathrm{TMS}$ 为内标, $\mathrm{CDCl}_{3}$ 为溶剂)测得; 比旋光度测试于美国 Perkin Elmer 公司 Polarmetermodel 341 型旋光仪; 元素分析使用德国 Elementar 公司 Vario EL III 型元素分析仪测得; 熔点测试使用予华 X-5 熔点 测试仪; 数均分子量和分子量分布指数测试使用美国 Waters 1515 凝胶渗透色谱仪 (以聚乙烯为标准物质, 流 速 $1.0 \mathrm{~mL} / \mathrm{min}$ ); 产品 $e e$ 值经高效液相色谱分析: Dionex P680 HPLC, 流动相为正己烷和异丙醇, 手性柱 OD-H (Daicel Chemical Ind., Ltd, $25 \mathrm{~cm} \times 0.46 \mathrm{~cm}$ ), 柱温 $25{ }^{\circ} \mathrm{C}$, 流速 $1.0 \mathrm{~mL} / \mathrm{min}$. $(S)-2,2^{\prime}$-二羟基-1,1'-联薬来自 阿拉丁试剂公司, 实验中的四氢呋喃、甲苯等溶剂经钠/ 二苯甲酮干燥，二氯甲烷经氢化钲干燥后使用，所有反 应均在氩气保护下进行, 手性聚合物配体 Poly-A 和 Poly-B 参考文献[26]合成.

\section{2 实验方法}

\section{2 .1 苯乙炔和醛的不对称加成反应过程}

氩气氛围下，苯乙炔 $(220 \mu \mathrm{L}, 2.0 \mathrm{mmol})$ 和 1.0 $\mathrm{mol} \cdot \mathrm{L}^{-1}$ 的二甲基锌 $(2.0 \mathrm{~mL}, 2.0 \mathrm{mmol})$ 的甲苯溶液回流 5 $\mathrm{h}$, 生成白色固体. 冷却至室温后, 依次加入聚合物配体 (基于单体单元)(37.25 mg, $0.1 \mathrm{mmol}) 、$ 钛酸异丙酯 $(0.3$ $\mathrm{mL}, 1.0 \mathrm{mmol}), 15 \mathrm{~min}$ 后加入苯甲醛 $(51 \mu \mathrm{L}, 0.5 \mathrm{mmol})$. 反应 $24 \mathrm{~h}$ 后, 饱和氯化铵淬灭反应, 分离有机层, 水层 用 $10 \mathrm{~mL}$ 乙酸乙酯萃取. 合并有机相并用无水硫酸镁干 燥, 有机相过滤后减压浓缩并用薄层色谱 $[V$ (石油醚) : $V($ 乙酸乙酯 $)=8: 1]$ 纯化得到炔醇产物.

\subsection{2 (S)-2-羟基-2'-特戊酰氧基-1, $1^{\prime}$-联菜(2)的合成}

在 $0{ }^{\circ} \mathrm{C}$ 条件下, 往溶有 $(S)-2,2^{\prime}$-二羟基-1,1'-联菜 $(\mathbf{1})$
$(11.46 \mathrm{~g}, 40 \mathrm{mmol})$ 和三乙胺 $(16.8 \mathrm{~mL}, 120 \mathrm{mmol})$ 的乙腈 $(80 \mathrm{~mL})$ 溶液中逐滴滴加特戊酰氯 $(4.88 \mathrm{~g}, 40.4 \mathrm{mmol})$ 的 乙腈 $(40 \mathrm{~mL})$ 溶液. 滴加完毕后, 将反应混合物升温至 $25{ }^{\circ} \mathrm{C}$ 并且在该温度下反应 $6 \mathrm{~h}$. 反应结束后将反应混合 物减压浓缩除去溶剂后用乙醚 $(100 \mathrm{~mL})$ 溶解，再将混合 溶液依次用 $1 \mathrm{~mol} \cdot \mathrm{L}^{-1} \mathrm{HCl}(20 \mathrm{~mL} \times 2)$ 以及饱和食盐水 $(30 \mathrm{~mL} \times 2)$ 洗涤、无水硫酸镁干燥. 将有机相过滤, 滤液 减压蒸馏除去溶剂后所得残余物经柱层析 ( $V$ (石油醚) : $V($ 二氯甲烷 $)=3: 1$ ) 分离获得 $13.40 \mathrm{~g}$ 白色固体 $\mathbf{2}^{[53]}$, 产 率 90\%. m.p. 62 65 ${ }^{\circ} \mathrm{C}$ (文献值 ${ }^{[53]}$ m.p. 63 65 ${ }^{\circ} \mathrm{C}$ ); $[\alpha]_{\mathrm{D}}^{25}-56.4$ (c 0.5, THF); ${ }^{1} \mathrm{H}$ NMR $\left(\mathrm{CDCl}_{3}, 400 \mathrm{MHz}\right) \delta$ : 8.10 (d, $J=8.8 \mathrm{~Hz}, 1 \mathrm{H}), 8.01$ (d, $J=8.1 \mathrm{~Hz}, 1 \mathrm{H}), 7.91$ (d, $J=8.9 \mathrm{~Hz}, 1 \mathrm{H}), 7.83(\mathrm{~d}, J=7.9 \mathrm{~Hz}, 1 \mathrm{H}), 7.52(\mathrm{t}, J=6.4$ $\mathrm{Hz}, 1 \mathrm{H}), 7.40 \sim 7.31(\mathrm{~m}, 6 \mathrm{H}), 7.08(\mathrm{~d}, J=8.2 \mathrm{~Hz}, 1 \mathrm{H})$, $5.16(\mathrm{~s}, 1 \mathrm{H}), 0.79(\mathrm{~s}, 9 \mathrm{H}) ;{ }^{13} \mathrm{C} \mathrm{NMR}\left(\mathrm{CDCl}_{3}, 100 \mathrm{MHz}\right) \delta$ : $177.78,151.85,148.37,133.71,133.57,132.23,130.67$, $130.27,129.08,128.33,127.91,127.44,126.66,126.18$, $125.68,124.60,123.52,123.08,121.85,118.27,114.28$, 38.77, 26.49. Anal. calcd for $\mathrm{C}_{25} \mathrm{H}_{22} \mathrm{O}_{3} \mathrm{C} 81.06, \mathrm{H}$ 5.99; found C 81.05, H 5.96 .

3.2.3 (S)-6-溴-2-羟基-2'-特戊酰氧基-1, 1'-联菜 (3) 的 合成

在 $0{ }^{\circ} \mathrm{C}$ 条件下, 往溶有 $(S)$-2-羟基-2'-特戊酰氧基1,1'-联萘 $(2)$ (29.64 g, $80 \mathrm{mmol})$ 的乙腈 $(200 \mathrm{~mL})$ 溶液中缓 慢滴加 $\mathrm{Br}_{2}(10 \mathrm{~mL}, 195.5 \mathrm{mmol})$, 滴加完后，反应混合物 在 $0{ }^{\circ} \mathrm{C}$ 继续反应 $10 \mathrm{~h}$. 往反应混合物中加入 $100 \mathrm{~mL}$ $\mathrm{Na}_{2} \mathrm{~S}_{2} \mathrm{O}_{4}(23 \mathrm{~g}, 132 \mathrm{mmol})$ 水溶液, 将混合物用 $\mathrm{CH}_{2} \mathrm{Cl}_{2}$ (60 $\mathrm{mL} \times 3)$ 萃取, 合并萃取液并将其用饱和食盐水 $(40$ $\mathrm{mL} \times 3)$ 洗涤、无水硫酸镁干燥. 有机相过滤后经减压蒸 馏除去溶剂, 所得剩余物经柱层析 $[V$ (二氯甲烷) $: V$ (石 油醚 $)=1: 2$ ] 分离纯化获得 $32.35 \mathrm{~g}$ 白色固体 $\mathbf{3}^{[53]}$, 产率 90\%. m.p. $79 \sim 81{ }^{\circ} \mathrm{C}$; $[\alpha]_{\mathrm{D}}^{25}+6.2$ (c 0.5, THF); ${ }^{1} \mathrm{H}$ NMR $\left(\mathrm{CDCl}_{3}, 400 \mathrm{MHz}\right) \delta: 8.07(\mathrm{~d}, J=8.8 \mathrm{~Hz}, 1 \mathrm{H}), 7.99(\mathrm{~m}$, 2H), 7.79 (d, $J=8.8 \mathrm{~Hz}, 1 \mathrm{H}), 7.51$ (td, $J=7.2,1.2 \mathrm{~Hz}$, $1 \mathrm{H}), 7.25 \sim 7.40(\mathrm{~m}, 5 \mathrm{H}), 6.92(\mathrm{~d}, J=8.8 \mathrm{~Hz}, 1 \mathrm{H}), 5.22(\mathrm{~s}$, $1 \mathrm{H}), 0.81(\mathrm{~s}, 9 \mathrm{H}) ;{ }^{13} \mathrm{C} \mathrm{NMR}\left(\mathrm{CDCl}_{3}, 100 \mathrm{MHz}\right) \delta: 177.89$, $152.26,148.37,133.43,132.28,132.25,131.05,130.17$, $129.98,129.96,129.43,128.49,127.67,126.55,126.40$, $125.48,122.48,121.91,119.52,117.38,114.56,38.85$, 26.59. Anal. calcd for $\mathrm{C}_{25} \mathrm{H}_{21} \mathrm{BrO}_{3} \mathrm{C} 66.82, \mathrm{H}$ 4.71; found C 66.85, H 4.75 .

\subsection{4 (S)-6-溴-2,2'-二羟基-1,1'-联菜(4)的合成}

在 $\mathrm{Ar}$ 氛围下, 往溶有 $(S)$-6-溴-2-羊基-2'-特戊酰氧 基-1,1'-联菜 $(3)$ (28.0 g, $62.4 \mathrm{mmol})$ 的 THF (100 mL)溶液 中一次性加入 $\mathrm{KOH}(10.4 \mathrm{~g}, 184 \mathrm{mmol})$ 和 $\mathrm{H}_{2} \mathrm{O}(80 \mathrm{~mL})$, 
将反应混合物在 $30{ }^{\circ} \mathrm{C}$ 反应 $20 \mathrm{~h}$. 将反应混合物通过减 压浓缩除去溶剂, 所得剩余物用 $50 \mathrm{~mL}$ 乙酸乙酯溶解, 分离出有机相, 并将有机相依次用 $1 \mathrm{~mol} \cdot \mathrm{L}^{-1} \mathrm{HCl}(30$ $\mathrm{mL} \times 2)$ 和饱和食盐水 $(15 \mathrm{~mL} \times 2)$ 洗涤、无水硫酸镁干燥. 有机相过滤后减压浓缩除去溶剂获得 $22.30 \mathrm{~g}$ 白色固体 $4^{[53]}$, 产率 98\%. m.p. $83 \sim 86{ }^{\circ} \mathrm{C}$ (文献值 ${ }^{[53]}$ m.p. 83 $\left.85{ }^{\circ} \mathrm{C}\right) ;[\alpha]_{\mathrm{D}}^{25}+6.5$ (c $\left.0.5, \mathrm{THF}\right) ;{ }^{1} \mathrm{H}$ NMR $\left(\mathrm{CDCl}_{3}, 400\right.$ MHz) $\delta: 7.01(\mathrm{~d}, J=8.0 \mathrm{~Hz}, 1 \mathrm{H}), 7.09(\mathrm{~d}, J=7.2 \mathrm{~Hz}, 1 \mathrm{H})$, $7.31 \sim 7.42(\mathrm{~m}, 5 \mathrm{H}), 7.39(\mathrm{t}, J=5.8 \mathrm{~Hz}, 1 \mathrm{H}), 7.86 \sim 7.90$ $(\mathrm{m}, 2 \mathrm{H}), 7.98(\mathrm{~d}, J=8.6 \mathrm{~Hz}, 1 \mathrm{H}), 8.17(\mathrm{~s}, 1 \mathrm{H}) ;{ }^{13} \mathrm{C} \mathrm{NMR}$ $\left(\mathrm{CDCl}_{3}, 100 \mathrm{MHz}\right) \delta: 153.16,152.87,133.49,132.22$, $131.75,131.01,130.82,130.74,130.48,129.63,128.62$, $127.78,126.29,124.30,124.17,119.06,118.01,117.96$, 111.58, 110.46. Anal. calcd for $\mathrm{C}_{20} \mathrm{H}_{13} \mathrm{BrO}_{2} \mathrm{C} 65.77, \mathrm{H}$ 3.59; found C 65.75 , H 3.58 .

3.2.5 (S)-6-激-2, $2^{\prime}$-二甲氧甲氧基-1, $1^{\prime}$-联蔡(5)的合成 在氩气保护以及 $0{ }^{\circ} \mathrm{C}$ 条件下, 往 $\mathrm{NaH}(3.88 \mathrm{~g}, 80$ $\mathrm{mmol})$ 和 THF $(80 \mathrm{~mL})$ 的混合物中缓慢滴加溶有 $(S)-6$ 溴-2,2'-二羟基-1,1'-联菜(4) (14.58 g, $40 \mathrm{mmol}$ )的 THF $(80 \mathrm{~mL})$ 溶液. $30 \mathrm{~min}$ 后往上述混合物中缓慢滴加氯甲基 甲醚 $(6.4 \mathrm{~g}, 80 \mathrm{mmol})$, 滴加完后将混合物在常温下反应 $6 \mathrm{~h}$. 加入 $30 \mathrm{~mL}$ 水淬灭反应, 分离出有机层, 水相用乙 酸乙酯 $(40 \mathrm{~mL} \times 2)$ 萃取, 合并有机相并用无水硫酸镁干 燥. 将有机相过滤, 滤液减压浓缩除去溶剂获得的剩余 物经柱层析分离获得 $16.28 \mathrm{~g}$ 白色固体 $\mathbf{5}^{[54]}$, 产率 $90 \%$. m.p. $78 \sim 79{ }^{\circ} \mathrm{C}$ (文献值 ${ }^{[54]}$ m.p. $78 \sim 79{ }^{\circ} \mathrm{C}$ ); $[\alpha]_{\mathrm{D}}^{25}-59.6$ (c 0.01, THF); ${ }^{1} \mathrm{H} \mathrm{NMR}\left(\mathrm{CDCl}_{3}, 400 \mathrm{MHz}\right) \delta: 8.04$ (s, $\left.1 \mathrm{H}\right)$, $7.95(\mathrm{~d}, J=9.0 \mathrm{~Hz}, 1 \mathrm{H}), 7.87(\mathrm{t}, J=8.2 \mathrm{~Hz}, 2 \mathrm{H}), 7.63 \sim$ $7.56(\mathrm{~m}, 2 \mathrm{H}), 7.36(\mathrm{t}, J=7.3 \mathrm{~Hz}, 1 \mathrm{H}), 7.29(\mathrm{~s}, 1 \mathrm{H}), 7.24$ (d, $J=7.1 \mathrm{~Hz}, 1 \mathrm{H}), 7.11(\mathrm{~d}, J=8.4 \mathrm{~Hz}, 1 \mathrm{H}), 7.04(\mathrm{~d}, \quad J=9.0$ $\mathrm{Hz}, 1 \mathrm{H}), 5.08$ (t, $J=6.0 \mathrm{~Hz}, 2 \mathrm{H}), 4.98(\mathrm{~d}, J=6.1 \mathrm{~Hz}, 2 \mathrm{H})$, 3.15 (s, $6 \mathrm{H}) ;{ }^{13} \mathrm{C}$ NMR $\left(\mathrm{CDCl}_{3}, 100 \mathrm{MHz}\right) \delta$ : 153.07, $152.76,133.94,132.64,131.01,129.93,129.81,129.64$, $129.57,128.45,127.95,127.50,126.45,125.32,124.15$, $121.68,120.62,118.38,117.95,117.23,95.32,95.24$, 55.84. Anal. calcd for $\mathrm{C}_{24} \mathrm{H}_{21} \mathrm{BrO}_{4} \mathrm{C}$ 63.59, $\mathrm{H}$ 4.67; found C 63.51, H 4.71.

3.2.6 (S)-6-甲酰基- $2,2^{\prime}$-二甲氧甲氧基-1, $1^{\prime}$-联菜 $(6)$ 的 合成

在氩气保护以及 $-78{ }^{\circ} \mathrm{C}$ 条件下, 将 $n$-BuLi $(2.5$ $\left.\mathrm{mol} \cdot \mathrm{L}^{-1}, 24.0 \mathrm{~mL}, 60 \mathrm{mmol}\right)$ 的正已烷溶液逐滴滴加到 (S)-6-澳-2,2'-二甲氧基甲氧基-1,1'-联萗(5) (18.14 g, 40 $\mathrm{mmol})$ 的 THF $(100 \mathrm{~mL})$ 的溶液中, 滴加完毕后, 保持混 合物在该温度下继续反应 $1 \mathrm{~h}$. 在 $-78{ }^{\circ} \mathrm{C}$ 下将 $\mathrm{DMF}$ $(10.2 \mathrm{~mL}, 128.2 \mathrm{mmol})$ 缓慢滴加到上述混合物中, 滴加
完后将混合物在 $-78{ }^{\circ} \mathrm{C}$ 反应 $3 \mathrm{~h}$. 将温度升至 $-20{ }^{\circ} \mathrm{C}$, 加入冰水 $(30 \mathrm{~mL})$ 用于淬灭反应, 将混合物减压蒸除溶 剂后所得剩余物用二氯甲烷 $(50 \mathrm{~mL})$ 溶解, 并往混合物 中加入 $50 \mathrm{~mL}$ 水, 分离出有机相后水相用二氯甲烷(30 $\mathrm{mL} \times 2)$ 萃取, 合并有机相, 依次用饱和食盐水 $(30 \mathrm{~mL} \times$ 2)洗涤有机相, 有机相经无水硫酸镁干燥后过滤除去干 燥剂, 滤液减压浓缩除去溶剂得到的剩余物经柱层析 $[V$ (石油醚) $: V($ 乙酸乙酯 $)=6: 1$ ]分离纯化获得 $10.40 \mathrm{~g}$ 白色固体 6 $^{[55]}$, 产率 $65 \%$. m.p. 95 98 ${ }^{\circ} \mathrm{C} ;[\alpha]_{\mathrm{D}}^{25}-56.4(c$ $0.01, \mathrm{THF}) ;{ }^{1} \mathrm{H} \mathrm{NMR}\left(\mathrm{CDCl}_{3}, 400 \mathrm{MHz}\right) \delta: 10.10(\mathrm{~s}, 1 \mathrm{H})$, 8.38 (s, 1H), 8.13 (d, $J=9.1 \mathrm{~Hz}, 1 \mathrm{H}), 7.98$ (d, $J=9.1 \mathrm{~Hz}$, $1 \mathrm{H}), 7.91(\mathrm{~d}, J=8.2 \mathrm{~Hz}, 1 \mathrm{H}), 7.71(\mathrm{~d}, J=9.1 \mathrm{~Hz}, 2 \mathrm{H}), 7.61$ (d, $J=9.1 \mathrm{~Hz}, 1 \mathrm{H}), 7.36$ (t, $J=7.2 \mathrm{~Hz}, 1 \mathrm{H}), 7.24$ (d, $J=9.0$ $\mathrm{Hz}, 1 \mathrm{H}), 7.12(\mathrm{~d}, J=8.4 \mathrm{~Hz}, 1 \mathrm{H}), 5.17(\mathrm{~d}, J=6.9 \mathrm{~Hz}, 1 \mathrm{H})$, $5.11(\mathrm{~d}, J=6.8 \mathrm{~Hz}, 1 \mathrm{H}), 5.07(\mathrm{~d}, J=6.9 \mathrm{~Hz}, 1 \mathrm{H}), 4.98(\mathrm{~d}$, $J=6.7 \mathrm{~Hz}, 1 \mathrm{H}), 3.18(\mathrm{~d}, J=17.6 \mathrm{~Hz}, 6 \mathrm{H}) ;{ }^{13} \mathrm{C}$ NMR $\left(\mathrm{CDCl}_{3}, 100 \mathrm{MHz}\right) \delta$ : 192.12, 155.44, 152.71, 137.51, $134.81,133.84,132.65,131.29,129.90,128.81,128.15$, $126.66,126.60,125.15,124.26,123.33,121.53,120.17$, 117.62, 116.98, 95.06, 94.64, 56.04, 55.90. Anal. calcd for $\mathrm{C}_{25} \mathrm{H}_{22} \mathrm{O}_{5}$ C 74.62, H 5.53; found C 74.58, H 5.49.

3.2.7 (S)-6-差䍩甲基- $2,2^{\prime}$-二甲氧甲氧基- $1,1^{\prime}$-联䓠(7)的 合成

冰浴下将 $(S)$-6-甲酰基-2,2'-二甲氧甲氧基-1, 1 '-联荎 (6) $(9.25 \mathrm{~g}, 23 \mathrm{mmol})$ 充分溶解于 THF $(100 \mathrm{~mL})$ 中, 分批 次加入 $\mathrm{NaBH}_{4}(1.73 \mathrm{~g}, 46 \mathrm{mmol})$. 反应体系升至室温反 应一个小时后将反应混合物倾倒入 $100 \mathrm{~mL}$ 水中, 用乙 酸乙酯萃取混合物, 获得的有机相用无水硫酸镁干燥. 有机相过滤后减压浓缩得获得 $9.21 \mathrm{~g}$ 无色液体 $\mathbf{7}^{[26]}$, 产 率 99\%. $[\alpha]_{\mathrm{D}}^{20}-58.2$ (c 0.05, THF); ${ }^{1} \mathrm{H}$ NMR $\left(\mathrm{CDCl}_{3}, 400\right.$ MHz) $\delta: 3.05$ (s, 3H), $3.06(\mathrm{~s}, 3 \mathrm{H}), 4.71(\mathrm{~s}, 2 \mathrm{H}), 4.88(\mathrm{~d}$, $J=6.7 \mathrm{~Hz}, 2 \mathrm{H}), 4.96(\mathrm{~d}, J=3.4 \mathrm{~Hz}, 1 \mathrm{H}), 4.99$ (d, $J=3.4$ $\mathrm{Hz}, 1 \mathrm{H}), 7.02 \sim 7.17(\mathrm{~m}, 4 \mathrm{H}), 7.23(\mathrm{t}, J=6.8 \mathrm{~Hz}, 1 \mathrm{H}), 7.46$ (d, $J=9.0 \mathrm{~Hz}, 2 \mathrm{H}), 7.76(\mathrm{~s}, 1 \mathrm{H}), 7.78(\mathrm{~d}, J=8.0 \mathrm{~Hz}, 1 \mathrm{H})$, $7.82(\mathrm{~d}, J=5.6 \mathrm{~Hz}, 1 \mathrm{H}), 7.87(\mathrm{~d}, J=5.6 \mathrm{~Hz}, 1 \mathrm{H}) ;{ }^{13} \mathrm{C}$ NMR $\left(\mathrm{CDCl}_{3}, 100 \mathrm{MHz}\right) \delta: 55.81,65.42,95.10,95.21$, $117.29,117.38,121.10,121.22,123.90,125.30,125.58$, $125.57,125.91,126.20,127.71,129.27,129.28,129.69$, $129.68,133.39,133.81,136.29,152.50,152.61$. Anal. calcd for $\mathrm{C}_{25} \mathrm{H}_{24} \mathrm{O}_{5}$ C 74.24, H 5.98; found $\mathrm{C} 74.26, \mathrm{H}$ 6.01 .

$3.2 .8(S, S) /(S, R)-6$-(2,3-环氧丙氧基)甲基-2,2'-二(甲 氧基甲氧基)-1, $1^{\prime}$-联菜 $(\mathbf{8 a} / \mathbf{8 b})$ 的合成

将 $(S)$-6- 羟甲基-2,2'-二甲氧甲氧基-1,1'- 联萗 (7) $(4.05 \mathrm{~g}, 10 \mathrm{mmol})$ 溶解于无水的 THF $(30 \mathrm{~mL})$ 中, 随后往 
反应体系中加入粉末状氢氧化钾(400 mol $\%$ )和四丁基溴 化铵 $(10 \mathrm{~mol} \%)$. 反应混合物在室温下搅拌 $2 \mathrm{~h}$, 将 $(S)$-环 氧氯丙烷 $(200 \mathrm{~mol} \%$ ) 滴加到反应中, 反应继续在室温下 进行 $12 \mathrm{~h}$ 后结束. 往反应混合物中加入 $(40 \mathrm{~mL})$ 乙酸乙 酯并用食盐水洗涤有机相. 分离出的有机相用无水硫酸 镁干燥, 有机相过滤后减压浓缩除去溶剂, 初产物用柱 色谱分离纯化. 获得 $3.78 \mathrm{~g}$ 无色黏状液体 $\mathbf{8 a}^{[26]}$, 产率 $82 \%$. $[\alpha]_{365}^{20}-154.5$ (c 0.1, THF); ${ }^{1} \mathrm{H}$ NMR $\left(\mathrm{CDCl}_{3}, 400\right.$ MHz) $\delta: 7.96 \sim 7.92(\mathrm{~m}, 2 \mathrm{H}, \mathrm{ArH}), 7.88 \sim 7.83(\mathrm{t}, J=3.0$ $\mathrm{Hz}, 2 \mathrm{H}, \mathrm{ArH}), 7.58 \sim 7.56(\mathrm{~d}, J=9.0 \mathrm{~Hz}, 2 \mathrm{H}, \mathrm{ArH}), 7.35 \sim$ $7.33(\mathrm{t}, J=8.0 \mathrm{~Hz}, 1 \mathrm{H}, \mathrm{ArH}), 7.22 \sim 7.12(\mathrm{~m}, 4 \mathrm{H}, \mathrm{ArH})$, $5.08 \sim 5.05\left(\mathrm{~m}, 2 \mathrm{H}, \mathrm{CH}_{2} \mathrm{O}\right), 4.98 \sim 4.96\left(\mathrm{~m}, 2 \mathrm{H}, \mathrm{CH}_{2} \mathrm{O}\right)$, $4.70 \sim 4.67\left(\mathrm{dd}, J=2.4,6.0 \mathrm{~Hz}, 2 \mathrm{H}, \mathrm{CH}_{2}\right), 3.77 \sim 3.76(\mathrm{dd}$, $J=2.8,5.4 \mathrm{~Hz}, 1 \mathrm{H}, \mathrm{CH}), 3.49 \sim 3.47\left(\mathrm{~m}, 1 \mathrm{H}, \mathrm{CH}_{2}\right), 3.18$ (s, $\left.1 \mathrm{H}, \mathrm{CH}_{2}\right), 3.15$ (s, $\left.3 \mathrm{H}, \mathrm{CH}_{3}\right), 3.14$ (s, $\left.3 \mathrm{H}, \mathrm{CH}_{3}\right), 2.79 \sim$ $2.78\left(\mathrm{t}, J=3.2 \mathrm{~Hz}, 1 \mathrm{H}, \mathrm{CH}_{2}\right), 2.61\left(\mathrm{~s}, 1 \mathrm{H}, \mathrm{CH}_{2}\right) ;{ }^{13} \mathrm{C} \mathrm{NMR}$ $\left(\mathrm{CDCl}_{3}, 100 \mathrm{MHz}\right) \delta: 152.92,152.72,129.38,126.69$, $126.26,125.99,125.56,124.07,117.65,117.45,95.38$, 73.44, 70.89, 55.80, 50.84, 44.30. Anal. calcd for $\mathrm{C}_{28} \mathrm{H}_{28} \mathrm{O}_{6}$ C 73.03, H 6.13; found C 73.08, H 6.16.

经过上述同样的方法, $(S, R)$-6-(2,3-环氧丙氧基)甲

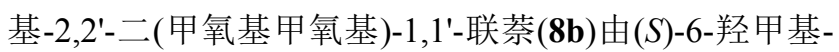
$2,2^{\prime}$-二甲氧甲氧基- $1,1^{\prime}$-联荎(7)和 $(R)$-环氧氯丙烷反应制 备, 获得 $3.68 \mathrm{~g}$ 淡黄色黏状液体 $8 \mathbf{b}$, 产率 $80 \%$. [ $[\alpha]_{365}^{20}-$ 102.4 (c 0.1, THF); ${ }^{1} \mathrm{H}$ NMR $\left(\mathrm{CDCl}_{3}, 400 \mathrm{MHz}\right) \delta: 7.96 \sim$ $7.92(\mathrm{~m}, 2 \mathrm{H}, \mathrm{ArH}), 7.88 \sim 7.83(\mathrm{t}, J=3.2 \mathrm{~Hz}, 2 \mathrm{H}, \mathrm{ArH})$, $7.59 \sim 7.57(\mathrm{~d}, J=9.0 \mathrm{~Hz}, 2 \mathrm{H}, \operatorname{ArH}), 7.35 \sim 7.33(\mathrm{t}, \quad J=$ $8.0 \mathrm{~Hz}, 1 \mathrm{H}, \mathrm{ArH}), 7.22 \sim 7.12(\mathrm{~m}, 4 \mathrm{H}, \mathrm{ArH}), 5.08 \sim 5.06$ $\left(\mathrm{m}, 2 \mathrm{H}, \mathrm{CH}_{2} \mathrm{O}\right), 4.98 \sim 4.96\left(\mathrm{~m}, 2 \mathrm{H}, \mathrm{CH}_{2} \mathrm{O}\right), 4.71 \sim 4.68$ (dd, $J=2.4,6.0 \mathrm{~Hz}, 2 \mathrm{H}, \mathrm{CH}_{2}$ ), $3.79 \sim 3.77$ (dd, $J=2.8,5.4$ $\mathrm{Hz}, 1 \mathrm{H}, \mathrm{CH}), 3.50 \sim 3.48\left(\mathrm{~m}, 1 \mathrm{H}, \mathrm{CH}_{2}\right), 3.17\left(\mathrm{~s}, 1 \mathrm{H}, \mathrm{CH}_{2}\right)$, $3.16\left(\mathrm{~s}, 3 \mathrm{H}, \mathrm{CH}_{3}\right), 3.15\left(\mathrm{~s}, 3 \mathrm{H}, \mathrm{CH}_{3}\right), 2.79 \sim 2.78(\mathrm{t}, J=3.2$ $\left.\mathrm{Hz}, 1 \mathrm{H}, \mathrm{CH}_{2}\right), 2.62\left(\mathrm{~s}, 1 \mathrm{H}, \mathrm{CH}_{2}\right) ;{ }^{13} \mathrm{C}$ NMR $\left(\mathrm{CDCl}_{3}, 100\right.$ MHz) $\delta: 152.85,152.75,134.07,133.67,129.97,129.76$, $129.38,127.86,126.68,126.27,125.99,125.57,124.07$, 117.55, 95.41, 73.44, 70.88, 55.81, 50.83, 44.30. Anal. calcd for $\mathrm{C}_{28} \mathrm{H}_{28} \mathrm{O}_{6}$ : C 73.03, H 6.13; found C 73.06, H 6.18.

3.2.9 $(S, S) /(S, R)$-6-(2,3-环氧丙氧基)甲基- $2,2^{\prime}$-二(甲 氧基甲氧基)-1, $1^{\prime}$-联菜的聚合以及脱 MOM 保护基过 程

将装有一定量的手性环氧单体 $(S, S) /(S, R)-6-(2,3-$-环 氧丙氧基)甲基- $2,2^{\prime}$-二 (甲氧基甲氧基)- $1,1^{\prime}$-联荎 (10 $\mathrm{mmol}$ )和 $1.5 \mathrm{~mL}$ 无水的二甲苯的聚合试管用氩气置换三 次, 在氩气氛围下加入 $0.34 \mathrm{mmol}$ 的颗粒状 $\mathrm{KOH}$. 将聚
合试管置于 $120{ }^{\circ} \mathrm{C}$ 的温度中摚拌 $20 \mathrm{~h}$. 在这过程中混合 物变得越来越粘稠, 颜色也越来越深. 将聚合试管冷却 至室温, 用 $10 \mathrm{~mL}$ THF 将聚合瓶中的混合物充分溶解, 将溶解液逐滴加入到大量的甲醇中 (溶液体积的 30 倍), 不断有白色固体析出, 抽滤分离得聚合物. 将聚合物用 $20 \mathrm{~mL}$ THF 溶解, $0{ }^{\circ} \mathrm{C}$ 下往溶解液中依次加入 $2 \mathrm{~mL}$ 的水 和 $2 \mathrm{~mL}$ 的浓盐酸. 混合物连续摚拌 $24 \mathrm{~h}$ 后逐滴加入到 搅拌的甲醇 $(200 \mathrm{~mL})$ 中, 不断有白色固体析出. 将白色 固体抽滤后反复沉降 3 次. 最后获得的聚合物置于 $50{ }^{\circ} \mathrm{C}$ 下真空干燥得白色固体 ${ }^{[26]}$, 产率 $82 \% \sim 85 \%$. ${ }^{1} \mathrm{H}$ NMR $\left(\mathrm{CDCl}_{3}, 400 \mathrm{MHz}\right) \delta$ : 8.02 6.76 [br, $\left.10 \mathrm{H}, \mathrm{Ar}-\mathrm{H}\right]$, $4.57 \sim 4.19\left[\mathrm{br}, 2 \mathrm{H}, \mathrm{CH}_{2} \mathrm{O}\right], 3.57 \sim 2.92[\mathrm{br}, 5 \mathrm{H},>\mathrm{CHO}$, $\left.\mathrm{CH}_{2}, \mathrm{CH}_{2} \mathrm{O}\right]$. Poly-A: 产率 85\%. [ $\left.\alpha\right]_{365}^{20}-342.4$ (c 0.05 , THF); $M_{\mathrm{n}}=5.2 \times 10^{3} ; \mathrm{PDI}=M_{\mathrm{w}} / M_{\mathrm{n}}=2.02$. Poly-B: 产率 $82 \%,[\alpha]_{365}^{20}-296.7$ (c $\left.0.05, \mathrm{THF}\right) ; M_{\mathrm{n}}=5.6 \times 10^{3} ; \mathrm{PDI}=$ $M_{\mathrm{w}} / M_{\mathrm{n}}=2.41$.

辅助材料(Supporting Information) 部分化合物的 ${ }^{1} \mathrm{H}$ NMR、 ${ }^{13} \mathrm{C}$ NMR 图谱以及催化产物的 HPLC 谱图. 这些 材料可以免费从本刊网站(http://sioc-journal.cn/)上下载.

\section{Referenes}

[1] Wu, Q.; Zhao, J.; Sun, S.; Tu, M.; Shi, F. Acta Chim. Sinica 2016, 74,576 (in Chinese).

(吴琼，赵佳佳，孙斯兵，屠蔓苏，石枫，化学学报，2016，74， 576.)

[2] Cheng, Q.; Xu, H.; Zhu, S.; Zhou, Q. Acta Chim. Sinica 2015, 73, 326 (in Chinese).

(程清卿, 许唤, 朱守非, 周其林, 化学学报, 2015, 73, 326.)

[3] Feng, X.; Du, H. Chin. J. Org. Chem. 2015, 35, 259 (in Chinese). (冯向青, 杜海峰, 有机化学, 2015, 35, 259.)

[4] Zhou, W.; Gao, L.; Tao, M.; Su, X.; Zhao, Q.; Zhang, J. Acta Chim. Sinica 2016, 74, 800 (in Chinese). (周伟，高利华，陶梦娜，宿晓，赵庆杰，张俊良，化学学报， 2016, 74, 800.)

[5] Ye, X.; Zeng, X.; Zhou, J. Acta Chim. Sinica 2016, 74, 984 (in Chinese). (叶旭, 曾兴平, 周剑, 化学学报, 2016, 74, 984.)

[6] Zheng, Y.; Ma, H.; Ma, J-A. Chin. J. Chem. 2016, 34, 511.

[7] Li, J.; Du, D. Chin. J. Chem. 2015, 33, 418.

[8] Liu, Y.; Zhou, J. Acta Chim. Sinica 2012, 70, 1451 (in Chinese). (刘运林, 周剑, 化学学报, 2012, 70, 1451.)

[9] Dupont, J.; de Souza, R. F.; Suarez, P. A. Z. Chem. Rev. 2002, 102, 3667.

[10] Fan, Q.-H.; Li, Y.-M.; Chan, A. S. C. Chem. Rev. 2002, 102, 3385.

[11] Song, C. E.; Lee, S.-G. Chem. Rev. 2002, 102, 3495.

[12] Bergbreiter, D. E.; Tian, J.; Hongfa, C. Chem. Rev. 2009, 109, 530.

[13] Fraile, J. M.; Garcia, J. I.; Herrerias, C. I.; Mayoral, J. A.; Pires, E. Chem. Soc. Rev. 2009, 38, 695.

[14] Fraile, J. M.; García, J. I.; Mayoral, J. A. Chem. Rev. 2009, 109, 360.

[15] Trindade, A. F.; Gois, P. M. P.; Afonso, C. A. M. Chem. Rev. 2009, $109,418$.

[16] Fan, Q. H.; Ren, C. Y.; Hu, W. H.; Chan, A. S. C. J. Am. Chem. Soc. 1999, 121, 7407.

[17] Fan, Q. H.; Chen, Y. M.; Chen, X. M.; Jiang, D. Z.; Xi, F.; Chan, A S. C. Chem. Commun. 2000, 31, 789. 
[18] Huang, W. S.; Hu, Q. S.; Pu, L. J. Org. Chem. 1999, 64, 7940.

[19] Hu, Q. S.; Pu, L. Polym. Prepr. 2000, 41, 16.

[20] Yang, X. W.; Sheng, J. H.; Da, C. S.; Wang, H. S.; Su, W.; Wang, R.; Chan, A. S. C. J. Org. Chem. 2000, 65, 295.

[21] Liu, D.; Zhu, W.; Zhang, A.; Yang, N.; Yang, L. Chin. J. Org. Chem. 2015, 35, 1797 (in Chinese). (刘大财, 朱维君, 张安林, 阳年发, 杨利文, 有机化学, 2015, 35, 1797.)

[22] Yan, J.; Yang, Z.; Yang, N. Chin. J. Org. Chem. 2016, 36, 812 (in Chinese).

(晏瑾懿，阳珠生，阳年发，有机化学, 2016, 36, 812.)

[23] Huang, C.; Yang, N.; Zhang, A.; Yang, L. Polymer 2012, 53, 3514.

[24] Zhang, A.; Yang, N.; Yang, L.; Peng, D. Chem. Lett. 2013, 43, 462.

[25] Chen, Y.; Qin, G.; Yang, L.; Yang, N. Chin. J. Chem. 2015, 33, 463.

[26] Zhang, A.-L.; Yu, Z.-d.; Yang, L.-W.; Yang, N.-F. Tetrahedron: Asymmetry 2015, 26, 173 .

[27] Zhang, A.-L.; Yu, Z.-D.; Yang, L.-W.; Yang, N.-F.; Peng, D. J. Mol. Catal. A: Chem. 2015, 398, 407.

[28] Liu, D.; Ouyang, K.; Yang, N. Tetrahedron 2016, 72, 1018.

[29] Corey, E. J.; Cimprich, K. A. J. Am. Chem. Soc. 1994, 116, 3151.

[30] Roush, W. R.; Sciotti, R. J. J. Am. Chem. Soc. 1994, 116, 6457.

[31] Stang, P. J.; Diederich, F. Modern Acetylene Chemistry, VCH, Weinheim, 1995.

[32] Myers, A. G.; Zheng, B. J. Am. Chem. Soc. 1996, 118, 4492.

[33] Thompson, A.; Corley, E. G.; Huntington, M. F.; Grabowski, E. J. J.; Remenar, J. F.; Collum, D. B. J. Am. Chem. Soc. 1998, 120, 2028.

[34] Fox, M. E.; Li, C.; Marino, J. P.; Overman, L. E. J. Am. Chem. Soc. 1999, $121,5467$.

[35] Trost, B.; Krische, M. J. J. Am. Chem. Soc. 1999, 121, 6131.

[36] Gao, G.; Moore, D.; Xie, R.-G.; Pu, L. Org. Lett. 2002, 4, 4143.
[37] Ishizaki, M.; Hoshino, O. Tetrahedron: Asymmetry 1994, 5, 1901.

[38] Anand, N. K.; Carreira, E. M. J. Am. Chem. Soc. 2001, 123, 9687.

[39] Lu, G.; Li, X.; Zhou, Z.; Chan, W. L.; Chan, A. S. C. Tetrahedron: Asymmetry 2001, 12, 2147.

[40] Pu, L.; Yu, H. B. Chem. Rev. 2001, 101, 757.

[41] Lu, G.; Li, X.; Chan, W. L.; Chan, A. S. Chem. Commun. 2002, 2, 172.

[42] Chen, Z.-C.; Hui, X.-P.; Yin, C.; Huang, L.-N.; Xu, P.-F.; Yu, X.-X.; Cheng, S.-Y. J. Mol. Catal. A: Chem. 2007, 269, 179.

[43] Jiang, B.; Chen, Z. L.; Huang, H. Lett. Org. Chem. 2005, 2, 319

[44] Wu, P.-Y.; Wu, H.-L.; Shen, Y.-Y.; Uang, B.-J. Tetrahedron: Asymmetry 2009, 20, 1837.

[45] Rachwalski, M.; Leśniak, S.; Kiełbasiński, P. Tetrahedron: Asymmetry 2010, 21, 2687.

[46] Bauer, T.; Smoliński, S.; Gaweł, P.; Jurczak, J. Tetrahedron Lett. 2011, 52, 4882.

[47] Marinova, M.; Kostova, K.; tzvetkova, P.; tavlinova-kirilova, M.; chimov, A.; nikolova, R.; shivachev, B.; dimitrov, V. Tetrahedron: Asymmetry 2013, 24, 1453.

[48] Chen, C.; Huang, Q.; Zou, S.; Wang, L.; Luan, B.; Zhu, J.; Wang, Q.; Pu, L. Tetrahedron: Asymmetry 2014, 25, 199.

[49] Rachwalski, M. Tetrahedron: Asymmetry 2014, 25, 219.

[50] Jarzyński, S.; Leśniak, S.; Pieczonka, A. M.; Rachwalski, M. Tetrahedron: Asymmetry 2015, 26, 35.

[51] Marshall, J. A.; Wang, X. J. J. Org. Chem. 1992, 57, 1242.

[52] Mao, J.; Bao, Z.; Guo, J.; Ji, S. Tetrahedron 2008, 64, 9901.

[53] Yuan, X. Y.; Li, H. Y.; Hodge, P.; Kilner, M.; Tastard, C. Y.; Zhang, Z. P. Tetrahedron Asymmetry 2006, 17, 2401.

[54] Wang, X.; Wang, X.; Guo, H.; Wang, Z.; \& Ding, K. Chem. Eur. J. 2005, 11,4078 .

[55] Liu, G. H.; Tang, W. J.; Fan, Q. H. Tetrahedron 2003, 59, 8603.

(Li, L.; Fan, Y.) 